\title{
A user manual to measure gas diffusion kinetics in plants: Pneumatron construction, operation and data analysis
}

1 C.L. Trabi ${ }^{1}$, L. Pereira ${ }^{1,2,3^{*}}$, X. Guan' ${ }^{1}$, M.T. Miranda ${ }^{2}$, P.R.L. Bittencourt ${ }^{4}$, R.S. Oliveira ${ }^{3}$, R.V.

2 Ribeiro $^{3}$ and S. Jansen ${ }^{1}$

$3{ }^{1}$ Institute of Systematic Botany and Ecology, Albert-Einstein-Allee 11, Ulm University, D-89081

4 Ulm, Germany

$5{ }^{2}$ Center R\&D in Ecophysiology and Biophysics, Agronomic Institute (IAC), Campinas, Brazil.

63 Department of Plant Biology, Institute of Biology, P.O. Box 6109, University of Campinas

7 (UNICAMP), Campinas 13083-970, Brazil.

$8{ }^{4}$ College of Life and Environmental Sciences, University of Exeter, Exeter, United Kingdom.

9 * Correspondence:

10 Luciano Pereira

11 biolpereira@gmail.com

13 Keywords: vulnerability to embolism, plant hydraulics, plant pneumatics, plant water relations, gas

14 flow, gas diffusion, xylem.

\section{Abstract}

17 The Pneumatron device presented measures gas diffusion kinetics in the xylem of plants. The device

18 provides an easy, low-cost, and powerful tool for research on plant water relations. Here, we describe

19 in detail how to construct and operate this device to estimate xylem vulnerability to embolism, and

20 how to analyse pneumatic data. Simple and more elaborated ways of constructing a Pneumatron are

21 shown, either using wires, a breadboard, or a printed circuit board. The instrument is based on an open-

22 source hardware and software system, which allows users to operate it in an automated or semi-

23 automated way. A step-by-step manual and a troubleshooting section are provided. An excel

24 spreadsheet and an R-script are also presented for fast and easy data analysis. This manual should help

25 new users to avoid common mistakes, especially regarding stable measurements of the minimum and

26 maximum amount of gas that can be discharged from xylem tissue. 


\section{Introduction}

The Pneumatron is a device that allows automated measurements of the gas diffusion kinetics in plant xylem tissue (Pereira et al., 2020a). Pneumatic measurements have been applied to xylem tissue of various plant organs, such as stems, roots, and leaves, to estimate vulnerability to hydraulic failure of the water conducting cells, which is especially relevant to plants that undergo drought stress. Although the device has been designed to address questions in the field of xylem anatomy and physiology, it is expected that the instrument can also be applied to a wide range of other porous media.

During a pneumatic method measurement, a partial vacuum is pulled in a discharge tube to extract gas from xylem tissue of a cut branch, petiole, or root during less than one minute (Pereira et al., 2016; Bittencourt et al., 2018). The amount of gas extracted can be calculated from pressure measurements, using the ideal gas law. The Pneumatron has been shown to provide a major improvement of the manual pneumatic apparatus because the vacuum condition, pressure sensor readings, data storage, and the opening or closing of valves can be done within milliseconds. By applying repetitive measurements over time, and combining these pneumatic data with a quantification of sample dehydration, a "vulnerability curve" can be obtained in a straightforward way. For a general understanding of the manual pneumatic method, we refer to literature (Pereira et al., 2016, 2020a; Bittencourt et al., 2018; Zhang et al., 2018; Jansen et al., 2020).

So far, pneumatic vulnerability curves have been conducted for hundreds of species by few research groups. Although its construction is simple and based on an accessible and open-source platform (Arduino), there is a need for a detailed manual with clear instructions on the construction of a Pneumatron device, its operation, and the analysis of pneumatic data. Such details are crucial to introduce new research group to pneumatic measurements, which are different from measurements of hydraulic conductivity, and to ensure accurate and correct interpreation of the data obtained. This paper aims to provide such a user manual, and will avoid common mistakes in pneumatic experiments and misinterpretation of measurements. Besides its importance for measuring xylem embolism resistance, the Pneumatron can also be used to study gas kinetics of plants in vivo, and vessel length distributions (Pereira et al., 2020b; Yang et al., Submitted). These methods require minor modifications of the software programme and tube connections. Contrary to other standard methods on embolism resistance, the Pneumatron device is very fast and user-friendly, which makes this device also useful for field measurements. 
61 The Pneumatron is composed of a microcontroller system (Atmega328P, Microchip, on anArduino®

62 Uno board), a data storage (SD card) and real time clock (DS1307, Maxim Integrated; both assembled

63 on an Adafruit ${ }^{\circledR}$ Data Logger Shield), a 16 bits analog-to-digial converter (ADS1115, Texas

64 Instrument), a vacuum pump, a solenoid valve (3/2 connection normal closed) and its driver (logic-

65 level N-channel mosfet plus flyback diode) (Fig. 1a). These components are easily available in 66 electronic shops.

67 A printed circuit board (PCB) to construct the Pneumatron Shield (Fig. 1b), may be used to facilitate

68 the connection among electronic parts, reducing connection problems and providing better electronic

69 properties while requiring minimal soldering skills. The PCB can be easily manufactured via several

70 PCB producers by using the production files (Gerber Files; available in

71 https://github.com/Pneumatron/construction). Alternatively, it is possible to use a breadboard to make

72 all connections (Fig.1c), although this is not recommended, as it increases the chance of failure due to

73 bad contacts and, if not properly done, can lead to voltage gradients in the analog-to-digital converter

74 and sensor supply and grounding. A breadboard is not a permanent option, but can be useful for testing

75 or for a low demand experiment.

76 The individual components of the Pneumatron are listed in Table S1. The assembly and testing do not

77 require specialized knowledge, but correct orientation of some components is must be respected (Fig

78 1a). For protecting the sensitive parts of the Pneumatron, it is advised to enclose these into a box. It is

79 recommended to do this for preventing any damage to the vacuum pump and solenoid valve and

80 potential short circuit.

81 After assembling the electronic parts (Fig. 1), the pressure sensor, the vacuum pump, and the solenoid

82 valve need to be connected to each other with silicone tubing (Fig. 2a). These components are then

83 connected to the PCB circuit board (Fig. 2a). Once you have assembled the pins onto the data logger

84 shield and installed the different components onto the PCB board, you need to assemble them into a

85 stacked-up structure with the Arduino at the bottom, the data logger shield in the middle, and the

86 Pneumatron shield at the top (Fig. 2b).

\section{$87 \quad 2.2$ Software}

88 The Pneumatron is built on an Arduino® Uno or similar board. To be able to upload or modify the programmes, installation of the software is required. Further information can be found on the Arduino web-site (https://www.arduino.cc/en/Main/Software). System information and requirements are needed to get the correct version of the programme, depending on which platform the software is to be installed on. 
93 In order to get the programme fully functioning, some of the libraries need to be installed or updated.

94 The libraries used in this version of the Pneumatron are the SPI and and Wire (v. 2.3.5) for SPI and

95 I2C communication, RTCLib (version 1.2.4) for using the DS1307 real-time clock,

96 Adafruit_ADS1X15 (v. 1.0.1) for using the ADS1115 analog-to-digital converter and the SD library

97 (v. 2.3.5) for using SD the card with FAT32 file system. More information can be found at:

98 https://www.arduino.cc/en/guide/libraries. Arduino novices might require further training on how to

99 upload a given programme onto the board. Some complementary information is available at:

100 https://www.arduino.cc/en/main/howto.

101 The programmes for the Pneumatron can be downloaded from

102 https:/github.com/Pneumatron/software. Once you have uploaded the programme, your equipment is

103 ready for calibration and testing.

\section{$104 \quad 2.3$ Programmes}

\section{$105 \quad$ 2.3.1 Automated mode}

106 The programme controls the pump and the solenoid valve, and reads data from the pressure sensor via

107 an ADC 16 bits converter (Fig. 3). Firstly, the pump will be heard creating a partial vacuum inside the

108 discharge tubing (ca. $40 \mathrm{kPa}$ of absolute pressure), and should achieve this partial vacuum in less than

109 one second. If not, either the vacuum pump is not working well, or there is a leakage. After reaching

110 the target pressure, it will stop and the pressure values will be recorded for one minute (this period can

111 be changed depending on the experimental requirements). During this measuring period, the LED will

112 flash every $0.5 \mathrm{~s}$. Finally, the apparatus pressure will return to atmospheric pressure via repeated

113 opening and closing of the solenoid valve over a short time period (5 s), and the device will then wait

114 for the next measurement to be taken (e.g., every $15 \mathrm{~min}$ ). The suggested minimum time interval

115 between measurements is $15 \mathrm{~min}$, which allows for the gas in the xylem tissue to reequilibrate with

116 atmospheric pressure before the next measurement.

\section{$117 \quad$ 2.3.2 Semi-automated mode}

118 The semi-automated mode is similar to the automated one, but each individual measurement has to be

119 acttivated by the operator through a button. The rest of the semi-automated process is similiar to the

120 automated method, except for activation of the solenoid valve at the end of the measurement. The

121 button to take a measurement can only be pressed after an entire measuring cycle has been finished.

\section{$123 \quad 3 \quad$ Methods}

\section{$124 \quad 3.1 \quad$ Testing and Calibration}




\section{$125 \quad 3.1 .1$ Testing the Pneumatron for leakage}

126 After assembling the Pneumatron, the device should be tested for leakage. This is achieved by blocking 127 off the tubing that is used for sample connection, while running the programme for several 128 measurement cycles in the semi-automated mode. If the pump is working permanently, or frequently 129 (e.g. more than once during a single measurement) please refer to the troubleshooting section (see the

130 results section). Then, the device is powered off and the SD card removed. An ideal and fast way to 131 test the data obtained is to import the csv file into excel and to select only the data column on the right,

132 plotting the gas extraction data into a scatter point graph. The data should be as close to constant as 133 possible (within 1 to $2 \%$ ), apart from the first point, which is close to zero. If the data seems to be 134 drifting more than 5\% and decreasing regularly with each measurement, there is a leakage. More 135 information about how to deal with leakages can be found in the troubleshooting section.

\section{$136 \quad 3.1 .2$ Calibration}

137 The Pneumatron measurements are based on relative values (percentage of air discharge) calculated

138 from the difference between the initial and the final pressure measured in the discharge tubing. For this

139 reason, the units used, that is volts or $\mathrm{kPa}$, are not critical. However, if the correct pressure unit is 140 required for a different purpose, it is necessary to calibrate the pressure sensor instead of using the 141 manufacturer equation (default in the Pneumatron programmes) since the relation voltage/pressure 142 changes depending on the voltage supply. To calibrate the sensor, use the calibration software 143 (https://github.com/Pneumatron/software). There are two easy methods for calibration, one relying on 144 the height of a water column (Fig. 4a), and the other one using a syringe pump (Fig. 4b).

\section{$145 \quad$ 3.1.2.1 Water column calibration}

146 The pressure sensor (disconnected from the valve and pumping system, and reconnected to the tube 147 with the syringe), the tubing, and the syringe should be fully filled with water. The syringe is then fixed 148 by the stand at a certain height. The distance between the pressure sensor and the water surface of the 149 liquid should then be measured. This process is repeated at two or three height levels.

150 When the measurements have been taken, the data should be extracted from the Pneumatron and the 151 TXT file imported in any data plotter software. The pressure measurements should be represented by 152 a step graph and the value for each step should be linked with the height of the water column. To 153 determine the pressure that is produced by the water column, the following equation is used: 
157 Where $\rho$ is the density of the liquid used $\left(1,000 \mathrm{~kg} \mathrm{~m}^{-3}\right.$ for water $), \mathrm{g}$ is the acceleration of gravity $(9.81$

$158 \mathrm{~m} \mathrm{~s}^{-2}$ ) and $\mathrm{h}$ is the height between the pressure sensor and the free surface of the liquid, in meter. The

159 resulting pressure will be in $\mathrm{Pa}$, and can be converted to $\mathrm{kPa}$ for ease of use $(1,000 \mathrm{~Pa}=1 \mathrm{kPa})$.

160 The calibration equation is obtained by determining fitting the applied pressure (y-axis) to the sensor

161 voltage (x-axis). This equation (the slope and intercept values) should then be included in the

162 programme (see instruction at the initial lines of the programme) and the programme uploaded once

163 more onto the Arduino board.

\section{$164 \quad 3.1 .2 .2$ Syringe pump calibration}

165 The Pneumatron is started and the air contained in the $60 \mathrm{~mL}$ syringe is compressed. The pressure measured is relative. When air in the $60 \mathrm{~mL}$ syringe is compressed to $50 \mathrm{~mL}$, the pressure is then 20

$167 \mathrm{kPa}$; at $40 \mathrm{~mL}$, the pressure is $50 \mathrm{kPa}$, and $100 \mathrm{kPa}$ is reached at $30 \mathrm{~mL}$. The same process is followed

168 as the water column calibration to determine the calibration equation and to upload the equation to the 169 board.

\section{$170 \quad$ 3.1.2.3 Implementation of the calibration parameters}

171 Depending on the manufacturer of the pressure sensor, the sensor response for a given pressure will be 172 different. In case the pressure sensor differs from the one suggested here, the datasheet should provide 173 information about the response of the sensor to the pressure applied, as well as about the power supplied 174 to the sensor, which is 10 volts in the Pneumatron. Please refer to the datasheet of the sensor and check 175 the equation to be implemented in the programme (see https://github.com/Pneumatron/software), as 176 shown in section 3.1.2.1.

\section{$178 \quad 3.2 \quad$ Experimental Techniques}

\section{$179 \quad$ 3.2.1 Sample preparation}

180 Unlike sampling for hydraulic measurements, plant material can be cut in air because the conduits that 181 are cut open need to be filled with air. The cut open conduits therefore represent an extension of the 182 discharge tube (Pereira et al., 2016; Jansen et al., 2020). The length of samples should be preferentially 183 longer than the maximum vessel length (Greenidge, 1952), which is unproblematic if terminal organs 184 such as terminal branches, roots, or single leaves are used as they are part of the end/start of the water 185 transport system, and they are not showing another cut. Plant samples, however, should be intact and 186 any damage due to natural or artificial wounds should be avoided. It is also possible to work with stem 187 or root segments, as long as air-entry is prevented at one of the cut sides (Wu et al., 2020). The distal end of a segment could, for instance, be blocked with super glue or a resin to avoid gas exchange. 
189 After cutting in air, samples should be covered up with a black plastic bag to avoid dehydration. Since

190 it is important to start pneumatic measurements on samples that are well hydrated, samples need to be

191 collected early in the morning, preferentially during the wet season. However, if these conditions are

192 not possible, samples can be left within the plastic bag with their cut ends in water for several hours or

193 one night before taking measurements.

\section{$194 \quad$ 3.2.2 Determination of the discharge tubing volume}

195 A crucial factor that determines the precision of the Pneumatron is the volume of the discharge tubing,

196 which needs to be proportional to the amount of gas discharged from the xylem (Pereira et al., 2020a).

197 To estimate the discharged tubing volume, you may use completely dehydrated organs, of similar size

198 of the sample of interest, to measure the maximum amount of gas that can be discharged. This step is

199 essential to prevent re-pumping when samples become considerably dehydrated, and to ensure

200 maximun resolution of the pressure sensor (Pereira et al., 2020a). Repetitive measurements are required

201 to make sure that the maximum amount of gas represents a constant plateau. The ideal volume of the

202 discharge tube can then be estimated by dividing the maximum volume of gas discharged in microliters

203 by 510.2 (Pereira et al., 2020a). It will give the volume in milliliters to maximize the Pneumatron 204 precision.

205 Alternatively, you may take a few measurements on the same dehydrated plant organ using a small 206 tubing volume $(<1 \mathrm{~mL})$, and check if the vacuum pump will restart during the measurement time (e.g. 207 within $1 \mathrm{~min}$ ). If yes, you must increase the tubing volume and test it again. This step needs to be 208 repeated until the pump is no longer reactivated within the $1 \mathrm{~min}$ interval. The volume can be adjusted 209 by adding or removing a certain amount of rigid tubing. It is important that the tubing has a constant

210 volume under the partial vacuum that is pulled. See Table S1 for tubing details. If the discharge tube

211 is not adjusted, the measuring error during pneumatic experiments can be considerably high (Jansen et 212 al., 2020; Pereira et al., 2021).

\section{$213 \quad$ 3.2.3 Always test for leakage before you start}

214 Close off the open tube of the Pneumatron, and insert an empty SD card to the data logger shield. 215 Connect the Pneumatron to a power supply system. If the software uploaded follows the automated 216 mode, you will immediately hear the vacuum pump. If not, you need to push the button to start the 217 measurement. Then, wait for one min until the first measurement cycle has finished. The LED light 218 flashes when pressure data are recorded, and stops when the measurement has finished. Then, remove 219 the SD card and check the data to see if there was any potential leakage during the measurement (See 220 details in how to get and analyse data, and the troubleshooting part for potential leakage). 


\subsubsection{Cleaning the memory card}

222 Because your data are always stored in the file "log.txt", it is important to clean the memory card before you start with new measurements. Make sure that the SD card has been inserted properly (correct orientation and tight insertion).

\section{3.2.5 Connecting a sample to the Pneumatron}

226 Using hydrated plant material, preferentially bagged to avoid rapid dehydration at the beginning, trim the cut end of the sample carefully with a fresh razor blade to have conduits nicely cut open. Cutting can be done in air as cut-open conduits should become embolised. When working with non-terminal branches, leaves, or roots, make sure that the length of the sample is longer than the maximum vessel length. Otherwise, a high amount of air will be sucked up via the cut-open conduit. As mentioned above, you also need to put glue at distal cuts or at broken twigs to avoid gas exchange via cut xylem. Altought it is possible that these cut or broken xylem structures are not connected to the cut-open xylem tissue to which the Pneumatron is connected, it is recommended to apply glue to these places. Connect the branch end to the Pneumatron with elastic tubing (see Table S1 for details of the tubing and clamps), and choose the best-fitting tube, considering the size of your cut sample end. Use parafilm, plastic clamps, or glue to ensure that there is the smallest possible leakage. should ideally be done under conditions of stable temperature and humidity. As initial dehydration is typically fast, samples can be bagged (or semi-bagged). This is especially recommended when working with species that are rather vulnerable to embolism, and will allow you to collect more data points with the Pneumatron, especially at water potentials that are not very negative.

3.2.6 Turning on the apparatus

243 Connect the apparatus to the power supply and the measurements will start automatically when following the automatic mode. Measurements will be taken at every 15 min by default, but the time interval can easily be changed in the programme. The calculation of the volume of air discharged for each measurement will be discussed below in the "Data analysis" section.

\subsubsection{Stopping the measurements}

248 Measurements can be stopped when the branches are completely dehydrated (see the next section about water potential measurements). After severe dehydration, the maximum amount of gas extracted from the plant sample has been achieved, and there will be no longer increases in the percentage of gas discharged with further dehydration. Then, a curve between air discharge and time or, preferentially 
253 A common mistake is that measurements are stopped too early, before the maximum amount of

254 extracted gas has been reached. In this case, the $\Psi_{50}$ (water potential in which $50 \%$ of xylem embolism

255 is formed) can be strongly underestimated as simulated in Figure 5. When working with a sample that

256 has leaves, it is recommended to run the measurements until the leaves become crispy. At this

257 dehydration point, it is also important to avoid leakages caused by stem shrinkage, tightening the clamp

258 and reapplying glue at the connection with the Pneumatron.

\section{$259 \quad 3.2 .8$ Taking water potential measurements}

260 When plotting vulnerability curves, xylem water potentials are required for the x-axis. Xylem water potential can be measured in different ways, with a pressure chamber or psychrometer.

\subsubsection{Water potential with pressure chamber}

263 The branches need to be bagged at least $30 \mathrm{~min}$ before the measurements. If the whole branch is put in a dark environment (black plastic bag), the xylem water potential of a branch should be in equilibrium with the leaf water potential. Then, leaf water potential is assumed to be the xylem water potential when using the pressure chamber.

Excise one to two leaves from the branch and measure the leaf water potential using a pressure chamber. After cutting the leaves from the branch, it is important to apply glue to seal the cuts, because even cut petioles may contain vessels that run directly into the stem xylem and could lead to artificial air entry. Then, the branches are left to dehydrate and new measurements are taken. Write down the water potential results and the exact time of the measurement, so that water potential data and pneumatic measurements can be matched later.

Although the Pneumatron generally takes measurements at 15 min time intervals, it is practically not feasible to take water potential measurements with a pressure chamber at such high frequency. Therefore, estimating the decline in xylem water potential over time by interpolation is recommended (Pereira et al., 2020a). Generally, water potential measurements should be taken more frequently during the start of the dehydration process, and longer time periods can be taken once stomata have closed and a linear decline in water potential has been obtained. Thus, usually five to ten measurements are enough to estimate the water potential decline over the entire dehydration period, depending also on the leaf availability.

\subsubsection{Water potential with stem/leaf psychrometer}

282 Leaf or stem psychrometers can be used, also in combination with pressure chamber measurements.

283 The great advantage of using psychrometers, is that once they have been installed, the water potential 284 is automatically monitored at a certain time interval, which could be set to the same time interval as the Pneumatron measurements. 


\section{Results}

\subsection{Data extraction and analysis of the vulnerability curves using the $R$ script}

The R script (https://github.com/Pneumatron/analysis) can be used to analyse the data. For this, the raw data from the Pneumatron (log.csv file) and another file with water potential data are needed, saved as a comma delimited file (csv). Headers of three columns must be defined: "date", "hour", and "psy" (date and hour, in the format of $\mathrm{dd} / \mathrm{mm} / \mathrm{yyyy}$ and hh:mm, respectively, and water potential measurements (psy)). Then, it is needed to input the experimental conditions as indicated at the beginning of the $\mathrm{R}$ script (file name and address, time for the initial and final pressures, reservoir volume, and atmospheric pressure). After running the script in $\mathrm{R}$, the water potential between two consecutive measurements will be estimated, assuming a linear decrease over time. For example, from six measured points during two days of branch dehydration (with three measurements at two hour intervals during early dehydration, and three measurements every five to eight hours), it will estimate the water potential every $15 \mathrm{~min}$, which corresponds to the same default time interval of Pneumatron measurements.

The script will also save three figures: the vulnerability curve (Fig. 6a), the volume of gas discharged versus water potential (Fig. 6b), and the volume of gas discharged versus time (Fig. 6c). By comparing the vulnerability curve (Fig. 6a) with the gas discharge curves (Fig. 6b and c), it is possible to infer problems regarding leakages or water potential measurements. Also, a file with the summarized results

will be saved (results.csv). The script estimates the $\Psi_{50}, \Psi_{88}$, and $\Psi_{12}$ by fitting a sigmoidal curve (p50.pad, p12.pad, and p88.pad results, following Pammenter and Vander Willigen (1998), or from the nearest data point measured directly by the Pneumatron (p50_near, p12_near, and p88_near results).

\subsection{Vulnerability curves using an Excel spreadsheet}

You may use the file data_example_pneumatron.xlsx to analyse the pneumatic data (Supplementary Material 2). A complete tutorial to use this excel file is shown in Supplementary Material 1. By curve fitting, vulnerability traits can be estimated in Excel, as shown in Supplementary Material 2.

\subsection{Troubleshooting}

During the construction and operation of Pneumatron devices, some of the following issues could be problematic. The most common problems are related to a leakage between the components, malfunctioning of data logger shield, or the solenoid valve. On the other hand, problems related to the Arduino board, electric components (such as Mosfets, resistors, etc.), or the vacuum pump are rare. 
- Check connections on the different shields and boards.

- Check that the pin connections are matching their counterparts.

- $\quad$ Check the pressure sensor and/or the ADC 16bits converter is accurately connected.

- Connect the Pneumatron to a computer, open the Arduino software and then open the serial monitor. Read the potential error messages if nothing appears to work (See section D). B. Frequent or continuous pumping of the vacuum pump (leakage)

- If the main source of leakage has been identified to be the solenoid valve, consider replacing it.

- An alternative, but uncommon source of leakage can be the T junction.

- A leakage may also come from a not accurately inserted tube. Check if the solenoid valve and the pump are properly connected (following the correct orientation).

- The pump screws may be loose, and thus the pump cannot create the pressure declared in the software.

C. No pumping at all when powered

- Check if the pressure is near zero and not between 10 and $50 \mathrm{kPa}$. The solenoid valve may not open properly at the end of a measurement and the pressure does not return to the atmospheric pressure. Consider increasing the tubing volume between the pump and solenoid valve or replace the solenoid.

- Check the connection to the pump.

- Check if the pressure sensor and/or the ADC 16bits converter are correctly connected.

- Check if the programme has been properly uploaded on the board.

D. No data recorded or unreasonable values (all identical values or outside range values)

- SD card may be set on locked, corrupted, unformatted, or not fully inserted in the data logger shield. Change to the unlocked position, format the card, or check if the card is fully inserted and in the right orientation.

- Check the pressure sensor and/or the ADC 16bits converter is correctly connected.

- There could be a problem with the data logger shield. The pins may be not well connected, or another problem that is hard to detect may occur. Consider replacing it.

- An error in the recording of time can be due to a different RTC (Real Time Clock), The programme has been develop with the RTC DS1307, but check the data logger. If the inscription PCF8523 is shown, you need to change RTC_DS1307 to RTC_PCF8523, and change rtc.isrunning by rtc.initialized.

E. The pump seems to run under powered 
- Check the power supply. The voltage or power might be too low. Consider replacing the power supply.

F. Arduino flashes rapidly but nothing else works

- This occurs when the voltage from the power supply is inferior to the optimal value. Consider changing the power supply.

- Alternatively, too many Mosfet transistors and/or too many pressure sensors are connected.

\section{Discussion}

360 The construction manual, the user manual details, and the software presented in this paper provide users with a Pneumatron device to estimate xylem embolism resistance. Vulnerability curves based on pneumatic measurements have been compared against various alternative methods (Pereira et al., 2016; Bittencourt et al., 2018; Zhang et al., 2018; Jansen et al., 2020). These earlier experiments showed that the pneumatic method provides accurate estimations of embolism resistance similar to the bench dehydration method, centrifuge-flow measurements (ChinaTron or cavitron; Cochard et al. (2005); Wang et al. (2014)), and the optical method (Brodribb et al., 2016). A comparison of $\Psi_{50}$ data based on the Pneumatic method with alternative approaches for 50 angiosperm species shows a strong and highly significant correlation $\left(\mathrm{R}^{2}=0.88\right.$, data not shown) considering published (Pereira et al., 2016, 2021; Zhang et al., 2018; Guan et al., 2020; Sergent et al., 2020) and unpublished results. Moreover, we used the Unit Pipe Pneumatic model to show that $91 \%$ of the gas volume extracted in $15 \mathrm{~s}$ of simulation comes from the first two series of intact and embolised vessels, while only $9 \%$ of the gas extracted results from xylem sap (Yang et al., submitted), which is saturated or supersaturated with gas (Schenk et al., 2016). This modelling exercise also showed that the Pneumatron may underestimate embolism resistance by 2 to $17 \%$, with a typical measuring error of $0.11 \mathrm{MPa}$ for $\Psi_{50}$ values. Such measuring accuracy is better or at least equal to the typical measuring disagreement by various hydraulic methods based on the same species (Cochard et al., 2013; Jansen et al., 2015).

The high measuring accuracy of the Pneumatron is mainly due to the fast axial diffusion of gas across intervessel pit membranes (Kaack et al., 2019), while radial diffusion across cell walls and between the xylem and the bark is extremely slow (Sorz and Hietz, 2006; Wang et al., 2015). Even if intervessel pit membranes are hydrated, with a characteristic water volume fraction of ca. 80\% (Zhang et al., 2020), diffusion across 200 to 1,000 nm thick pit membranes will be fast and within seconds (Yang et 
advantages over most conventional, both hydraulic and non-hydraulic methods (Table 1). The manual pneumatic approach, however, could result in a considerably larger measuring error due to unprecise recording of fast gas diffusion during the first seconds of gas extraction. Therefore, we recommend users to work with a Pneumatron device instead of the manual pneumatic approach, preferentially using the automated programme. In general, less data points are collected with the semi-automated mode and the connection and disconnection of samples may slightly change the leakage rates and tubing volume, which affects the measurement precision and the correct detection of the $\mathrm{GD}_{\min }$ and $\mathrm{GD}_{\max }$ plateaus (Fig. 4).

Yet, new users should be made well aware about the pneumatic principles, which are different from hydraulic measurements, and should be familiar with the basics that determine gas diffusion kinetics.

393 These physical laws include the ideal gas law, Henry's law for gas concentration partitioning between liquid and gas phases at equilibrium, and Fick's law for diffusion. When the Pneumatic method is incorrectly applied, data are likely misinterpreted (Chen et al., 2020; Sergent et al., 2020; Pereira et al., 2021). The two most common mistakes include incorrect adjustment of the discharge tube volume, and the lack of stable gas discharge measurements at the beginning and the end of pneumatic measurements $\left(G_{\min }\right.$ and $\left.\mathrm{GD}_{\max }\right)$. Since pneumatic vulnerability curves are normalised against $\mathrm{GD}_{\min }$ and $\mathrm{GD}_{\max }$, it is crucial to have reliable values for these two reference points (see Fig. 4). The consequences of not adjusting or incorrectly adjusting the volume of the discharge tube include a relatively high measuring error. When the discharge tube is too large or too small, the difference between the minimum and maximum air discharge volumes will be too small, resulting in relatively high measurement uncertainty (Jansen et al., 2020).

The main advantages of the Pneumatron device for constructing vulnerability curves are its low construction costs $(<€ 100)$, the easy, automated operation, the fast analysis of pneumatic data, and its measuring accuracy. These issues are especially relevant when measurements on a large number of samples or species are desired. The device can also be used under remote field conditions, although water potential measurements may then become more challenging than pneumatic measurements. Vessel dimensions do not affect the precision of embolism resistance based on pneumatic measurements, as long as the discharge tube volume has been adjusted properly. This means that the 412 more precise for wide vessels based on a Unit Pipe Pneumatic model (Yang et al., 2020). Ring-porous 413 species, however, may need to be tested carefully because earlywood vessels in growth rings that are 414 more than one year old are not only dysfunctional after one year, but also long and wide, and typically 415 plugged with tyloses (Sano et al., 2011). One solution to work with ring-porous species is to glue off 
416 the xylem of previous growth rings (Zhang et al., 2018). Species that secrete resin, mucilage, oil or

417 other substances, could also be problematic and should thus be tested carefully. Removing a thin slice

418 at the cut-open xylem between measurements may be a solution.

419 A disadvantage is that the method is destructive and requires cutting open xylem tissue. Unlike

420 hydraulic measurements, however, air entry of cut-open conduits should be aimed for as cut-open

421 conduits function as an extension of the discharge tube. In case xylem samples that are collected would

422 be under positive xylem pressure (Schenk et al., 2020), it is possible that xylem sap will come out at

423 the cut-open end, which will not only be problematic for pneumatic measurements, but also for

424 hydraulic methods. Other forms of wounding are likely slow and unlikely to affect the vessel

425 dimensions during dehydration. Special attention should also be paid to non-xylem tissue such as pith,

426 which may undergo some cracks and shrinkage during dehydration. Our experience, however, is that

427 cracks or shrinkage are frequently not problematic when samples with relatively small pith proportions

428 are used. Even if air from the pith tissue is extracted, this becomes only problematic when the gas

429 volume extracted from the pith increases considerably, which is unlikely due to slow diffusion to this

430 tissue. Also, cracks will only affect gas extraction amounts if these are connected to cut xylem conduits

431 or the cut xylem area. Another disadvantage is that the method has not been successfully applied yet

432 to gymnosperms. While the narrow tracheid dimensions could partially explain this, it is assumed that

433 the torus-margo pit membranes of conifers could result in fast aspiration of the pit membrane (Zelinka

434 et al., 2015; Schulte and Hacke, 2020), which prevents gas extraction from intact, embolised tracheids.

435 It is possible, however, that a modification of the applied vacuum, discharge tube volume, and/or

436 extraction time is needed to successfully apply the Pneumatron to gymnosperm xylem.

437 In addition to studies on xylem embolism, the Pneumatron has also been used to estimate vessel length

438 distribution in an easy and much faster way than conventional methods (Williamson and Milburn,

439 2017; Link et al., 2018; Pereira et al., 2020b). This is possible by an easy modification of the reservoir

440 volume and using the same software described here for the semi-automated mode. The data analysis is

441 also straightforward by using a specific R-script, and thus, it may pave the way for fast measurements

442 of vessel length distributions, and the non-random distribution of vessel ends near nodes, side branches,

443 stem-petioles transitions, etc. Pneumatic estimations of the hydraulically weighted vessel length have

444 been validated against the silicon-injection method for five species, and the air-injection method of

445 Cohen et al., (2003) for seven species (Yang et al., submitted).

446 We hope this manual makes the Pneumatron accessible to many plant biologists as a high quality, low-

447 cost, versatile tool. If required, the current version of the device can be modified and improved by

448 using updated or alternative hardware and software. Alternatively, a commercial and user-friendly 
version is already available (Plantem - Plant and Environment Technologies, Campinas, Brazil). Different versions have been used satisfactorily so far, using other components than proposed here (Pereira et al., 2020a; Wu et al., 2020), or increasing the number of samples that are simultaneously measured (Pereira et al., 2020a). Any modification of the hardware, such as the changes needed to allow simultaneous measurements on multiple samples, will require further adaptation to the software as well as the R-script for data analysis. We encourage future users to share modified and updated versions with other users in a collaborative spirit as this would further improve pneumatic measurements of xylem and avoid misinterpretation of data.

\section{Conflict of Interest}

459 The authors declare that the research was conducted in the absence of any commercial or financial 460 relationships that could be construed as a potential conflict of interest.

\section{$7 \quad$ Author Contributions}

463 CLT, LP, MTM, and XG wrote the first version of the manuscript. LP developed the R-script and the 464 Arduino software, this latter with contributions from CLT and PRLB. CLT created the calibration protocol, calibration software, and the troubleshooting section. XG and RVR wrote the protocol and the template for the Excel analysis. The manuscript received substantial contributions from SJ, RVR, PRLB, and RO.

\section{$8 \quad$ Funding}

470 The authors acknowledge funding from the German Research Foundation (SJ, project nr. 383393940 and nr. 410768178) and from the São Paulo Research Foundation (RVR, MTM, and LP, Grant \#2019/15276-8).

\section{Acknowledgments}

475 Financial support to SJ and XG is provided by a research grant from the German Research Foundation 476 (project nr. 383393940 and nr. 410768178). The authors acknowledge the São Paulo Research 477 Foundation (FAPESP, Brazil) for the research grant (RVR, LP and MTM, Grant 2019/15276-8), 478 fellowship (LP and RVR, Grant 2017/14075-3) and scholarship (MTM and RVR, Grant 2018/09834479 5). RVR are fellows of the National Council for Scientific and Technological Development (CNPq, 480 Brazil). 


\section{References}

Alder, N. N., Pockman, W. T., Sperry, J. S., and Nuismer, S. (1997). Use of centrifugal force in the study of xylem cavitation. J. Exp. Bot. 48, 665-674. doi:10.1093/jxb/48.3.665.

Bittencourt, P., Pereira, L., and Oliveira, R. (2018). Pneumatic method to measure plant xylem embolism. Bio-Protocol 8, 1-14. doi:10.21769/bioprotoc.3059.

Bonetti, S., Breitenstein, D., Fatichi, S., Domec, J. C., and Or, D. (2020). Persistent decay of fresh xylem hydraulic conductivity varies with pressure gradient and marks plant responses to injury. Plant Cell Environ., 1-16. doi:10.1111/pce.13893.

Bouda, M., Windt, C. W., McElrone, A. J., and Brodersen, C. R. (2019). In vivo pressure gradient heterogeneity increases flow contribution of small diameter vessels in grapevine. Nat. Commun. 10, 5645. doi:10.1038/s41467-019-13673-6.

Bréda, N., Cochard, H., Dreyer, E., and Granier, A. (1993). Field comparison of transpiration, stomatal conductance and vulnerability to cavitation of Quercus petraea and Quercus robur under water stress. Ann. des Sci. For. 50, 571-582. doi:10.1051/forest:19930606.

Brodribb, T. J., Skelton, R. P., Mcadam, S. A. M., Bienaimé, D., Lucani, C. J., and Marmottant, P. (2016). Visual quantification of embolism reveals leaf vulnerability to hydraulic failure. New Phytol. 209, 1403-1409. doi:10.1111/nph.13846.

Chen, Y.-J., Maenpuen, P., Zhang, Y.-J., Barai, K., Katabuchi, M., Gao, H., et al. (2020). Quantifing vulnerability to embolism in tropical trees and lianas using five methods: Can discrepancies be explained by xylem structural traits?. doi:10.1111/nph.16927.

Choat, B., Badel, E., Burlett, R., Delzon, S., Cochard, H., and Jansen, S. (2016). Noninvasive measurement of vulnerability to drought-induced embolism by X-Ray microtomography. Plant Physiol. 170, 273-282. doi:10.1104/pp.15.00732.

Cochard, H., Badel, E., Herbette, S., Delzon, S., Choat, B., and Jansen, S. (2013). Methods for measuring plant vulnerability to cavitation: A critical review. J. Exp. Bot. 64, 4779-4791. doi:10.1093/jxb/ert193.

Cochard, H., Cruiziat, P., and Tyree, M. T. (1992). Use of positive pressures to establish vulnerability curves: Further support for the air-seeding hypothesis and implications for pressure-volume

511 Cochard, H., Damour, G., Bodet, C., Tharwat, I., Poirier, M., and Améglio, T. (2005). Evaluation of a new centrifuge technique for rapid generation of xylem vulnerability curves. Physiol. Plant. 124, 
410-418. doi:10.1111/j.1399-3054.2005.00526.x.

514 Cohen, S., Bennink, J., and Tyree, M. (2003). Air method measurements of apple vessel length distributions with improved apparatus and theory. J. Exp. Bot. 54, 1889-1897. doi:10.1093/jxb/erg202.

De Baerdemaeker, N. J. F., Arachchige, K. N. R., Zinkernagel, J., Van Den Bulcke, J., Van Acker, J., the link between hydraulic conductivity and drought-induced embolism. Tree Physiol. 39, 16461664. doi:10.1093/treephys/tpz078.

Greenidge, K. N. H. (1952). An approach to the study of vessel length in hardwood species. Am. J. Bot. 39, 570-574. doi:10.1002/j.1537-2197.1952.tb13070.x.

Guan, X., Pereira, L., McAdam, S., Cao, K.-F., and Jansen, S. (2020). No gas source, no problem: preexisting embolism may affect non-pressure driven embolism spreading in angiosperm xylem by gas diffusion. Authorea Prepr., 1-18. doi:10.22541/au.159674582.24283294.

Gullo, M. A., and Salleo, S. (1992). Water storage in the wood and xylem cavitation in 1-year-old twigs of Populus deltoides Bartr. Plant, Cell Environ. 15, 431-438. doi:10.1111/j.13653040.1992.tb00993.x.

Hochberg, U., Albuquerque, C., Rachmilevitch, S., Cochard, H., David-Schwartz, R., Brodersen, C. R., et al. (2016). Grapevine petioles are more sensitive to drought induced embolism than stems: evidence from in vivo MRI and microcomputed tomography observations of hydraulic vulnerability segmentation. Plant. Cell Environ. 39, 1886-1894. doi:10.1111/pce.12688.

Jansen, S., Guan, X., Kaack, L., Trabi, C.L., Miranda, M. T., Ribeiro, R. V, et al. (2020). The Pneumatron estimates xylem embolism resistance in angiosperms based on gas diffusion kinetics: a mini-review. Acta Hortic. in press.

Jansen, S., Schuldt, B., and Choat, B. (2015). Current controversies and challenges in applying plant hydraulic techniques. New Phytol. 205, 961-964. doi:10.1111/nph.13229.

Kaack, L., Altaner, C. M., Carmesin, C., Diaz, A., Holler, M., Kranz, C., et al. (2019). Function and three-dimensional structure of intervessel pit membranes in angiosperms: a review. IAWA J. 40, 673-702. doi:10.1163/22941932-40190259.

Klepsch, M., Zhang, Y., Kotowska, M. M., Lamarque, L. J., Nolf, M., Schuldt, B., et al. (2018). Is xylem of angiosperm leaves less resistant to embolism than branches? Insights from microCT, hydraulics, and anatomy. J. Exp. Bot. 69, 5611-5623. doi:10.1093/jxb/ery321.

Link, R. M., Schuldt, B., Choat, B., Jansen, S., and Cobb, A. R. (2018). Maximum-likelihood estimation of xylem vessel length distributions. J. Theor. Biol. 455, 329-341. 
doi:10.1016/j.jtbi.2018.07.036.

Milburn, J., and Johnson, R. (1966). The conduction of sap. II. Detection of vibrations produced by sap cavitation in Ricinus xylem. Planta 69, 43-52.

Nolf, M., Beikircher, B., Rosner, S., Nolf, A., and Mayr, S. (2015). Xylem cavitation resistance can be estimated based on time-dependent rate of acoustic emissions. New Phytol. 208, 625-632. doi:10.1111/nph.13476.

Pammenter, N. W., and Van der Willigen, C. (1998). A mathematical and statistical analysis of the curves illustrating vulnerability of xylem to cavitation. Tree Physiol. 18, 589-593. doi:10.1093/treephys/18.8-9.589.

Peng, G., Yang, D., Liang, Z., Li, J., and Tyree, M. T. (2019). An improved centrifuge method for determining water extraction curves and vulnerability curves in the long-vessel species Robinia pseudoacacia. J. Exp. Bot. 70, 4865-4875. doi:10.1093/jxb/erz206.

Pereira, L., Bittencourt, P. R. L., Oliveira, R. S., Junior, M. B. M., Barros, F. V, Ribeiro, R. V., et al. (2016). Plant pneumatics: stem air flow is related to embolism - new perspectives on methods in plant hydraulics. New Phytol. 211, 357-370. doi:10.1111/nph.13905.

Pereira, L., Bittencourt, P. R. L., Pacheco, V. S., Miranda, M. T., Zhang, Y., Oliveira, R. S., et al. (2020a). The Pneumatron: An automated pneumatic apparatus for estimating xylem vulnerability to embolism at high temporal resolution. Plant. Cell Environ. 43, 131-142. doi:10.1111/pce.13647.

Pereira, L., Bittencourt, P. R. L., Rowland, L., Brum, M., Miranda, M. T., Pacheco, V. S., et al. (2021). Using the Pneumatic method to estimate embolism resistance in species with long vessels: A commentary on the article "A comparison of five methods to assess embolism resistance in trees." For. Ecol. Manage. 479, 118547. doi:10.1016/j.foreco.2020.118547.

Pereira, L., Miranda, M. T., Pires, G. S., Pacheco, V. S., Guan, X., Kaack, L., et al. (2020b). A semiautomated method for measuring xylem vessel length distribution. Theor. Exp. Plant Physiol. doi:10.1007/s40626-020-00189-4.

Sano, Y., Morris, H., Shimada, H., Ronse De Craene, L. P., and Jansen, S. (2011). Anatomical features associated with water transport in imperforate tracheary elements of vessel-bearing angiosperms. Ann. Bot. 107, 953-964. doi:10.1093/aob/mcr042.

Schenk, H. J., Espino, S., Visser, A., and Esser, B. K. (2016). Dissolved atmospheric gas in xylem sap measured with membrane inlet mass spectrometry. Plant Cell Environ. 39, 944-950. doi:10.1111/pce.12678.

Schenk, H. J., Jansen, S., and Hölttä, T. (2020). Positive pressure in xylem and its role in hydraulic 
function. New Phytol., nph.17085. doi:10.1111/nph.17085.

Schulte, P. J., and Hacke, U. G. (2020). Solid mechanics of the torus-margo in conifer inter-tracheid bordered pits. New Phytol. doi:10.1111/nph.16949.

Sergent, A. S., Varela, S. A., Barigah, T. S., Badel, E., Cochard, H., Dalla-Salda, G., et al. (2020). A comparison of five methods to assess embolism resistance in trees. For. Ecol. Manage. 468, 118175. doi:10.1016/j.foreco.2020.118175.

Sorz, J., and Hietz, P. (2006). Gas diffusion through wood: Implications for oxygen supply. Trees Struct. Funct. 20, 34-41. doi:10.1007/s00468-005-0010-x.

Sperry, J. S., Donnelly, J. R., and Tyree, M. T. (1988). A method for measuring hydraulic conductivity and embolism in xylem. Plant, Cell Environ. 11, 35-40. doi:10.1111/j.13653040.1988.tb01774.x.

Torres-Ruiz, J. M., Cochard, H., Choat, B., Jansen, S., López, R., Tomášková, I., et al. (2017). Xylem resistance to embolism: presenting a simple diagnostic test for the open vessel artefact. New Phytol. 215, 489-499. doi:10.1111/nph.14589.

Torres-Ruiz, J. M., Cochard, H., Mayr, S., Beikircher, B., Diaz-Espejo, A., Rodriguez-Dominguez, C. M., et al. (2014). Vulnerability to cavitation in Olea europaea current-year shoots: further evidence of an open-vessel artifact associated with centrifuge and air-injection techniques. Physiol. Plant. 152, 465-474. doi:10.1111/ppl.12185.

Tyree, M. T., Alexander, J., and Machado, J.-L. (1992). Loss of hydraulic conductivity due to water stress in intact juveniles of Quercus rubra and Populus deltoides. Tree Physiol. 10, 411-415. doi:10.1093/treephys/10.4.411.

Tyree, M. T., Dixon, M. A., Tyree, E. L., and Johnson, R. (1984). Ultrasonic Acoustic Emissions from the Sapwood of Cedar and Hemlock. Plant Physiol. 75, 988-992. doi:10.1104/pp.75.4.988.

Wang, Y., Burlett, R., Feng, F., and Tyree, M. T. (2014). Improved precision of hydraulic conductance measurements using a Cochard rotor in two different centrifuges. J. Plant Hydraul. 1, e007. doi:10.20870/jph.2014.e007.

Wang, Y., Liu, J., and Tyree, M. T. (2015). Stem hydraulic conductivity depends on the pressure at which it is measured and how this dependence can be used to assess the tempo of bubble pressurization in recently cavitated vessels. Plant Physiol. 169, 2597-2607. doi:10.1104/pp.15.00875.

Wheeler, J. K., Huggett, B. A., Tofte, A. N., Rockwell, F. E., and Holbrook, N. M. (2013). Cutting xylem under tension or supersaturated with gas can generate PLC and the appearance of rapid recovery from embolism. Plant, Cell Environ. 36, 1938-1949. doi:10.1111/pce.12139. 
612 Williamson, V. G., and Milburn, J. A. (2017). Xylem vessel length and distribution: Does analysis method matter? A study using Acacia. Aust. J. Bot. 65, 292-303. doi:10.1071/BT16220.

614 Wu, M., Zhang, Y., Oya, T., Marcati, C. R., Pereira, L., and Jansen, S. (2020). Root xylem in three 615 woody angiosperm species is not more vulnerable to embolism than stem xylem. Plant Soil 450, 479-495. doi:10.1007/s11104-020-04525-0.

617 Yang, D., Pereira, L., Peng1, G., Ribeiro, R. V., Kaack, L., Jansen, S., et al. (2020). A Unit Pipe 618 Pneumatic model to simulate gas kinetics during measurements of embolism in excised 619 angiosperm xylem. submitted.

620 Yin, P., and Cai, J. (2018). New possible mechanisms of embolism formation when measuring 621 vulnerability curves by air injection in a pressure sleeve. Plant. Cell Environ. 41, 1361-1368. doi:10.1111/pce.13163.

623 Zelinka, S. L., Bourne, K. J., Hermanson, J. C., Glass, S. V., Costa, A., and Wiedenhoeft, A. C. (2015). Force-displacement measurements of earlywood bordered pits using a mesomechanical tester. Plant, Cell Environ. 38, 2088-2097. doi:10.1111/pce.12532.

626 Zhang, Y., Carmesin, C., Kaack, L., Klepsch, M. M., Kotowska, M., Matei, T., et al. (2020). High porosity with tiny pore constrictions and unbending pathways characterize the 3D structure of intervessel pit membranes in angiosperm xylem. Plant Cell Environ. 43, 116-130. doi:10.1111/pce.13654.

Zhang, Y., Lamarque, L. J., Torres-Ruiz, J. M., Schuldt, B., Karimi, Z., Li, S., et al. (2018). Testing 


\section{Supplementary Material}

636 Supplementary Material 1 - Figure S1 - Schematic connection of the electronic components in a

637 Pneumatron device; Table S1 - Component list; Tutorial to use the excel file for data analysis.

638 Supplementary Material 2 - Excel file for data analysis.

\section{Data Availability Statement}

641 The datasets generated for this study can be found in the Github https://github.com/Pneumatron.

643 Table 1: Overview of the most common methods used to estimate xylem embolism resistance with the 644 technical advantages, disadvantages, and key-references.

\begin{tabular}{|c|c|c|c|}
\hline Method & Advantage & Disadvantage & Key references \\
\hline $\begin{array}{l}\text { Bench } \\
\text { dehydration }\end{array}$ & Cheap & $\begin{array}{l}\text { Time-consuming; plenty of plant } \\
\text { material needed; cutting-artefact; } \\
\text { wounding artefact; destructive }\end{array}$ & $\begin{array}{l}\text { (Sperry et al., 1988; Tyree et } \\
\text { al., 1992; Bréda et al., 1993; } \\
\text { Wheeler et al., 2013; De } \\
\text { Baerdemaeker et al., 2019; } \\
\text { Bonetti et al., 2020) }\end{array}$ \\
\hline $\begin{array}{l}\text { Centrifuge and } \\
\text { flow-centrifuge }\end{array}$ & $\begin{array}{l}\text { Fast; measurements rely on } \\
\text { water transport under negative } \\
\text { pressure }\end{array}$ & $\begin{array}{l}\text { Open-vessel artefact; expensive; } \\
\text { self-construction is technically } \\
\text { highly challenging; hydraulic } \\
\text { artefacts; destructive }\end{array}$ & $\begin{array}{l}\text { (Alder et al., 1997; Cochard et } \\
\text { al., 2005; Torres-Ruiz et al., } \\
\text { 2014, 2017; Wang et al., 2014; } \\
\text { Peng et al., 2019) }\end{array}$ \\
\hline $\begin{array}{l}\text { Air-injection } \\
\text { double end }\end{array}$ & Relatively fast & $\begin{array}{l}\text { Effervescence; vessel length } \\
\text { exceeds cavitation chamber } \\
\text { length; hydraulic artefacts; } \\
\text { destructive }\end{array}$ & $\begin{array}{l}\text { (Cochard et al., 1992; Gullo } \\
\text { and Salleo, 1992; Yin and Cai, } \\
\text { 2018) }\end{array}$ \\
\hline $\begin{array}{l}\text { Acoustic } \\
\text { emissions }\end{array}$ & $\begin{array}{l}\text { Rather expensive sensors; non- } \\
\text { destructive }\end{array}$ & $\begin{array}{l}\text { Data analysis time-consuming } \\
\text { and not straightforward }\end{array}$ & $\begin{array}{l}\text { (Milburn and Johnson, 1966; } \\
\text { Tyree et al., 1984; Nolf et al., } \\
\text { 2015) }\end{array}$ \\
\hline $\begin{array}{l}\text { Optical } \\
\text { vulnerability }\end{array}$ & $\begin{array}{l}\text { Cheap; applicable to leaf xylem } \\
\text { and the outermost xylem of } \\
\text { roots and stems; non-destructive }\end{array}$ & $\begin{array}{l}\text { Time-consuming image analysis; } \\
\text { mainly two-dimensional view of } \\
\text { embolism }\end{array}$ & $\begin{array}{l}\text { (Brodribb et al., 2016) } \\
\text { http://www.opensourceov.org/ }\end{array}$ \\
\hline MicroCT & Non-destructive imaging & $\begin{array}{l}\text { Extremely expensive; limited } \\
\text { beam-time availability; time- } \\
\text { consuming image analysis }\end{array}$ & $\begin{array}{l}\text { (Choat et al., 2016; Klepsch et } \\
\text { al., 2018) }\end{array}$ \\
\hline $\begin{array}{l}\text { Magnetic } \\
\text { resonance } \\
\text { imaging }\end{array}$ & $\begin{array}{l}\text { Non-destructive imaging, } \\
\text { quantification of flow }\end{array}$ & Low resolution; expensive & $\begin{array}{l}\text { (Hochberg et al., 2016; Bouda } \\
\text { et al., 2019) }\end{array}$ \\
\hline
\end{tabular}



available under aCC-BY-NC-ND 4.0 International license.

\section{5}

\begin{tabular}{|l|l|l|l|}
\hline Pneumatron & $\begin{array}{l}\text { Cheap and straightforward } \\
\text { operation, fast data analysis; } 1 \\
\text { sample = 1 vulnerability curve }\end{array}$ & $\begin{array}{l}\text { Adjustment of the discharge tube } \\
\text { volume; may not be applicable to } \\
\text { gymnosperms; destructive; no } \\
\text { visual, anatomical information }\end{array}$ & $\begin{array}{l}\text { (Pereira et al., 2016, 2020a; } \\
\text { Bittencourt et al., 2018; Zhang } \\
\text { et al., 2018) }\end{array}$ \\
\hline
\end{tabular}


bioRxiv preprint doi: https://doi.org/10.1101/2021.02.08,430283; this version posted February 10, 2021. The copyright holder for this preprint (which was not certified by peer review) is the author/funder, who has granted bioRxiv a license to display the preprint in perpetuity. It is made available under aCC-BY-NC-ND 4.0 International license.

647 Figure 1: Connection scheme between the Pneumatron components based on wires (a; the data logger 648 module, which is connected on top of the Arduino board, is not shown), a two-layer printed circuit 649 board (PCB) of a wireless Pneumatron shield (b) (https://github.com/Pneumatron/construction), and a 650 non-permanent assembly using a breadboard (c). The complete list of all components is listed in Table $651 \mathrm{~S} 1$ and a schematic connection among the parts is presented in Figure S1.

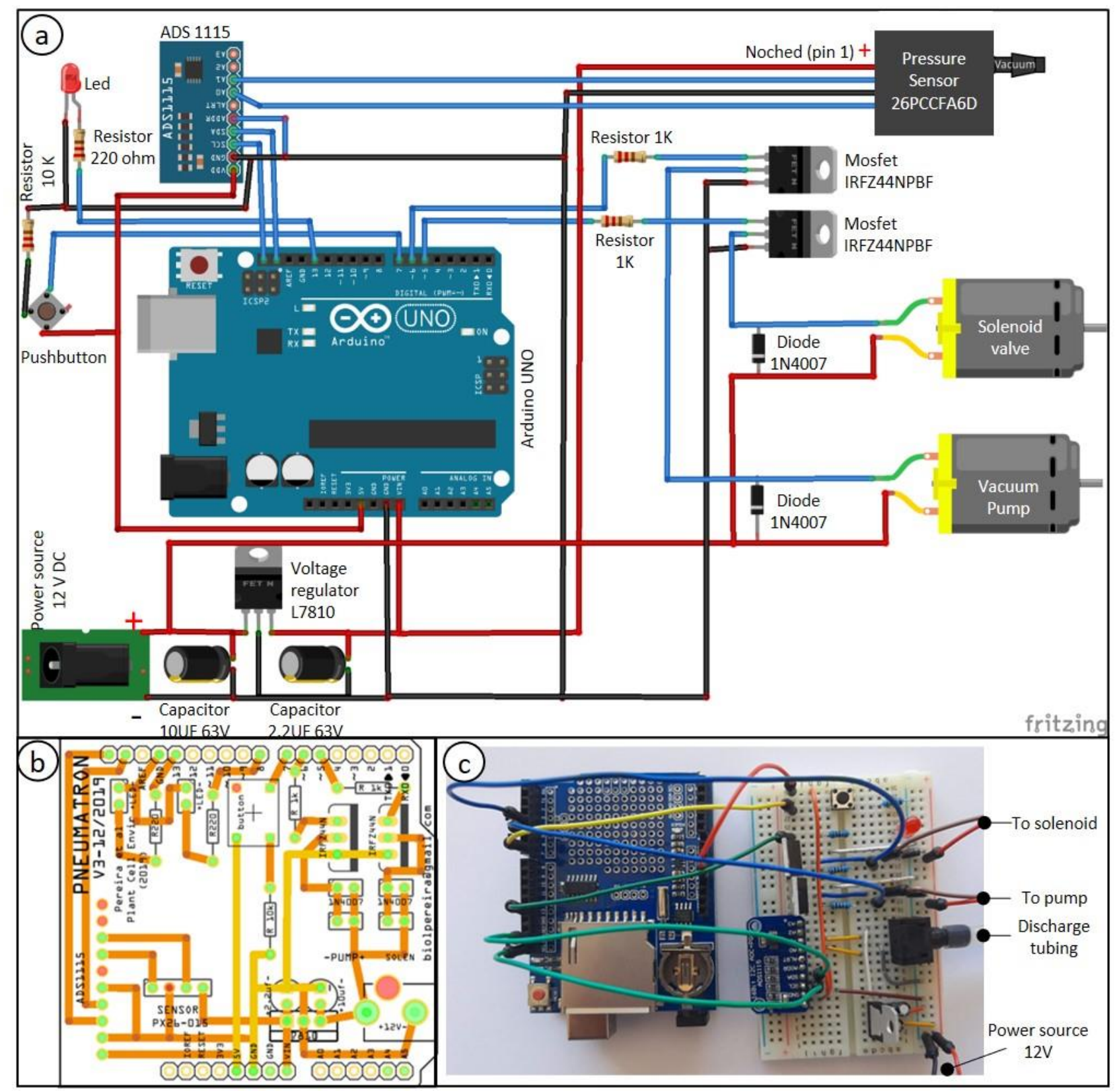


bioRxiv preprint doi: https://doi.org/10.1101/2021.02 08.430283; this version posted February 10,2021 . The copyright holder for this preprint (which was not certified by peer review) is the author/funder, who has granted bioRxiv a license to display the preprint in perpetuity. It is made available under aCC-BY-NC-ND 4.0 International license.

654 Figure 2: Components and connections (a) and assembling position (b) of the Pneumatron with a 655 Pneumatron shield (in which the push-button and power source were connected using wires).
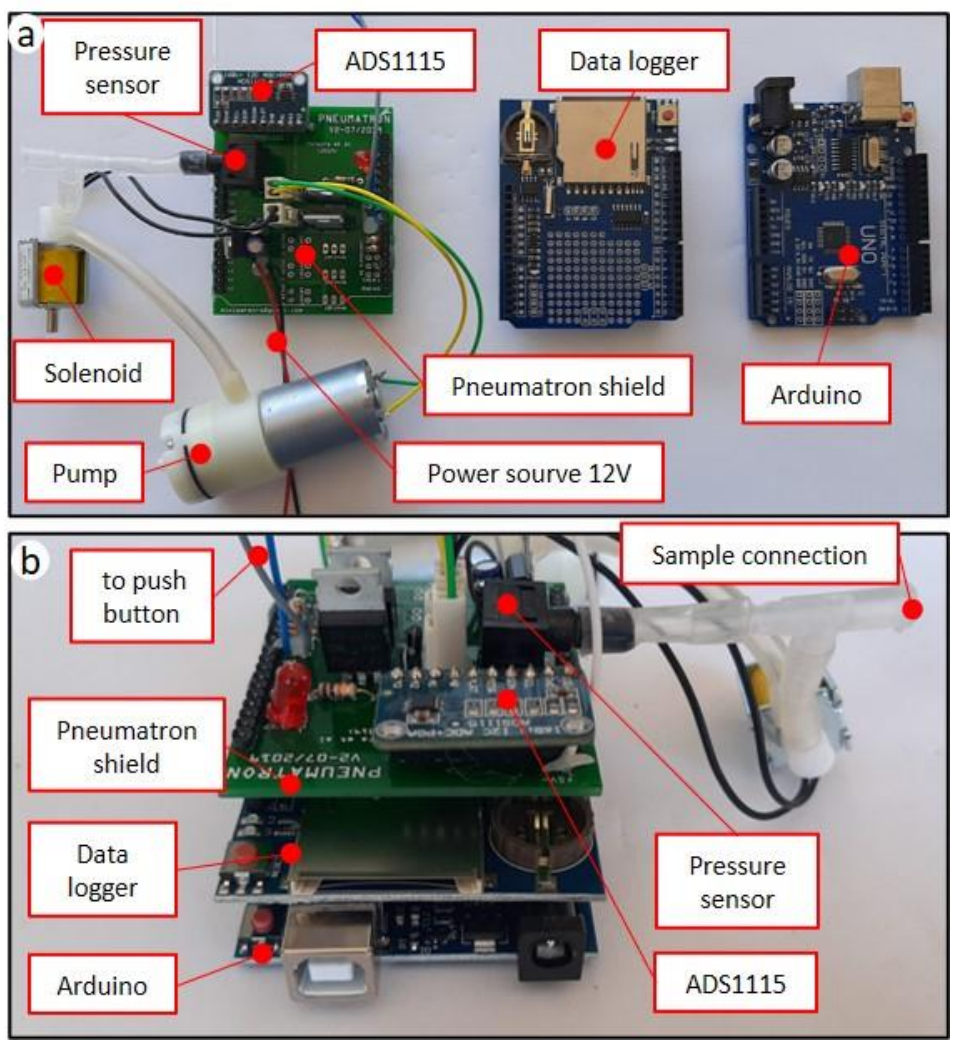
658 Figure 3: Workflow of a Pneumatron operation during automated mode.

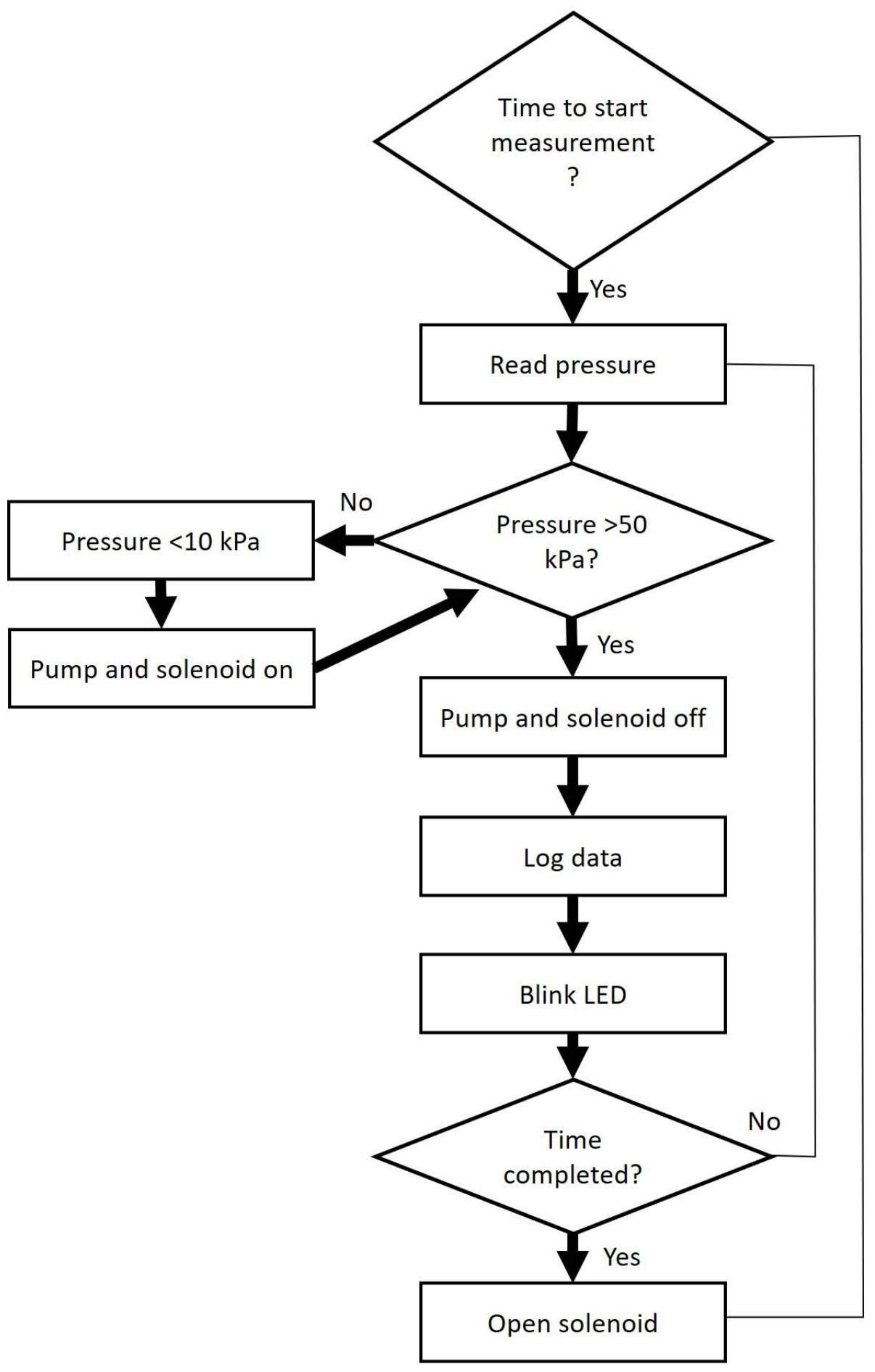


661 Figure 4: Calibration of the pressure sensor in a Pneumatron device using a water column (a), which 662 requires any container with known height of the water column (such as a syringe without the plunger), 663 tubing, a stand with a clamp to hold the container, and a ruler to measure the height of the water column 664 (from the pressure sensor to the free surface of the liquid). Calibration of a pressure sensor can also be 665 done using a syringe pump (b).
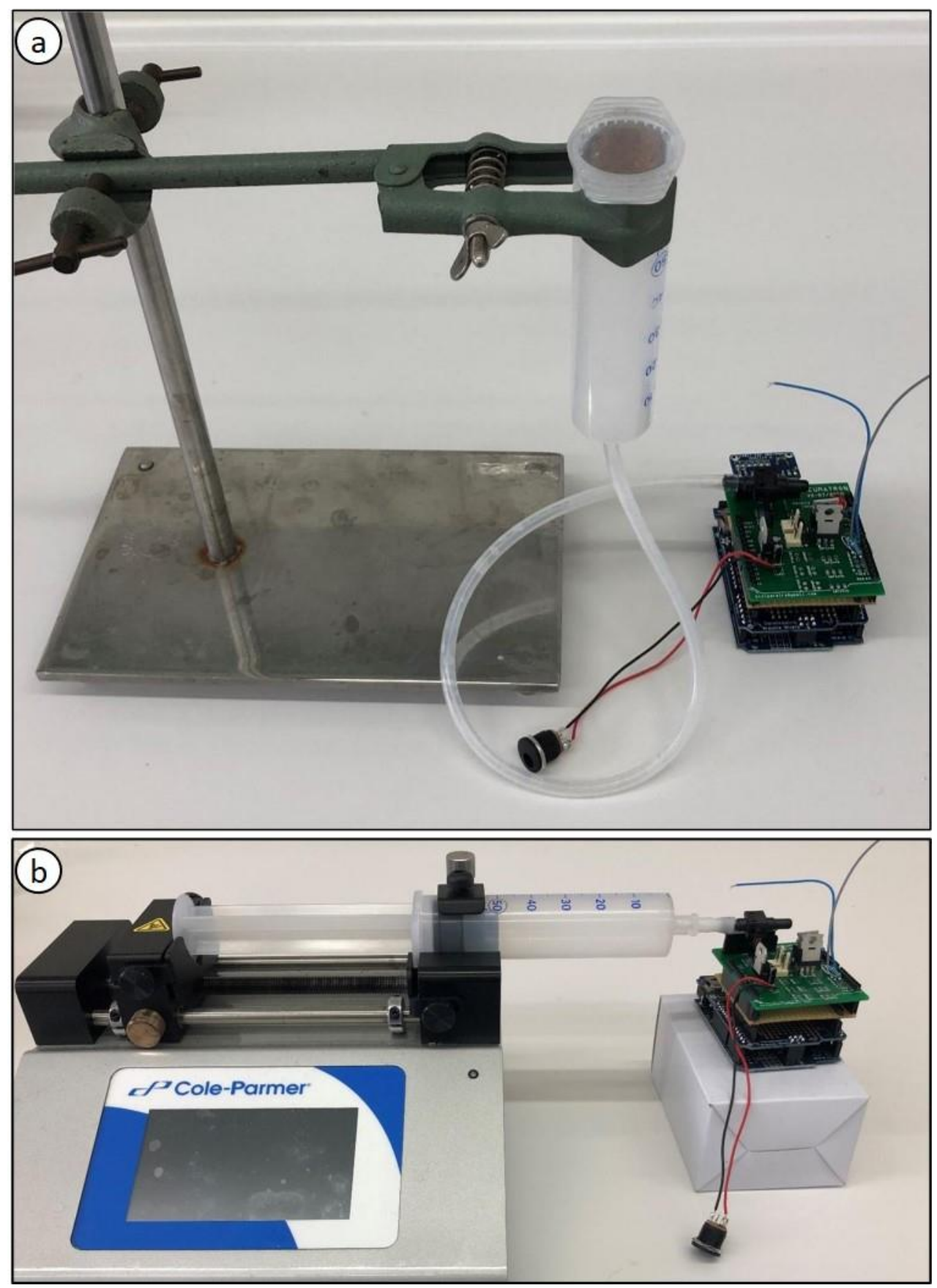
668 Figure 5: Vulnerability curves estimated with a Pneumatron for Olea europea, considering stable 669 maximum gas discharge $\left(\mathrm{GD}_{\max }\right)$ measurements (a plateau below -6 MPa, in blue), and using a 670 incorrect value as $\mathrm{GD}_{\max }$ (in red). The water potential at which $50 \%$ of gas discharge was estimated $671\left(\Psi_{50}\right.$, in $\left.\mathrm{MPa}\right)$ is shown with the same colors. The red curve and its $\Psi_{50}$ value should not be interpreted 672 as accurate measurements due to incorrect estimation of $\mathrm{GD}_{\max }$.

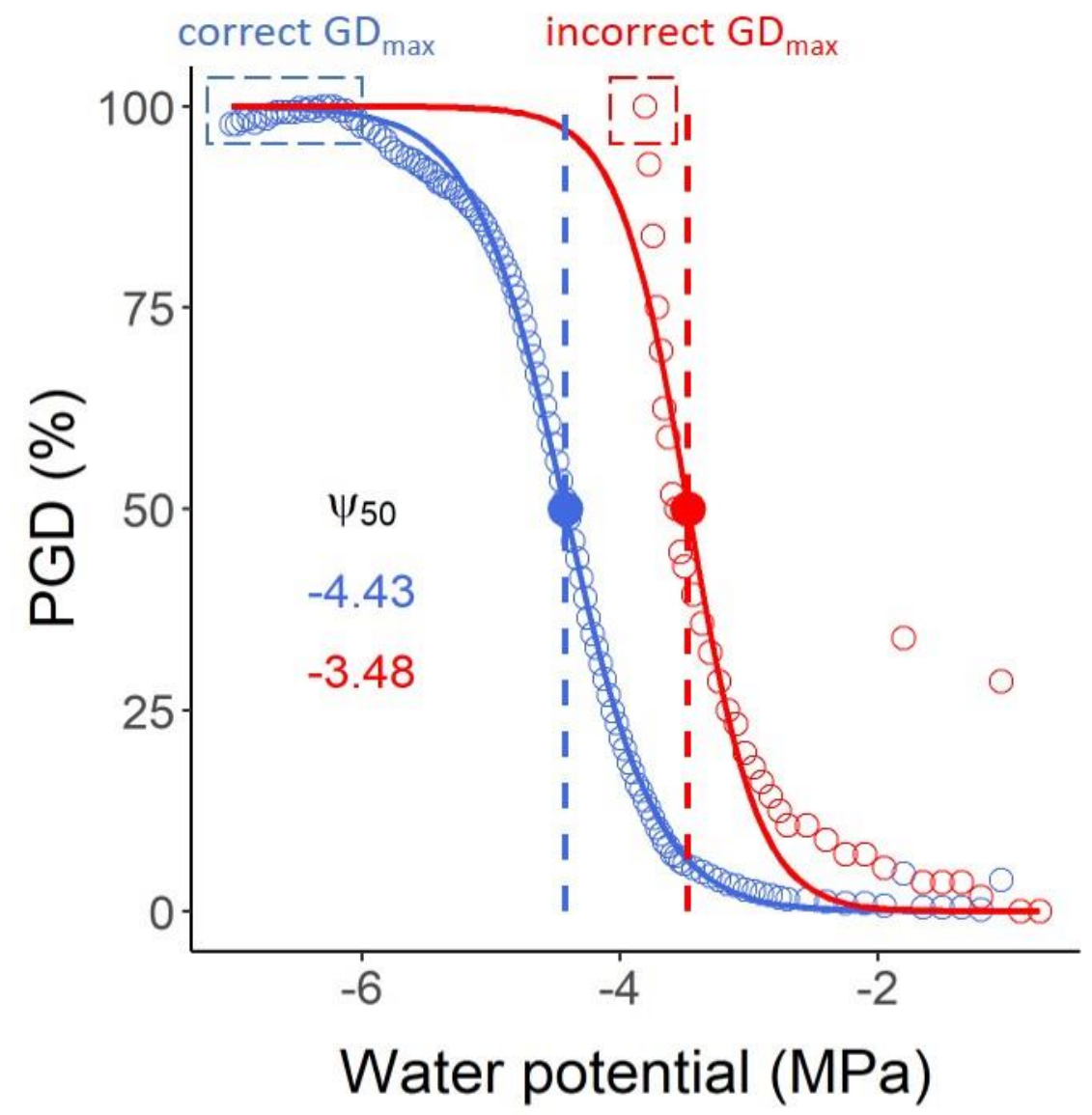


675 Figure 6: Example of output graphs from the R-script to analyse pneumatic gas discharge volumes

676 (GD) measured with a Pneumatron for Olea europea. In (a) the vulnerability curve and the traits

677 estimated from the sigmoidal curve (in blue) are shown. The values in red are the measured values at

$67812 \%, 50 \%$ and $88 \%$ PGD (percentage of gas discharge). The water potential corresponding to $50 \%$

679 PGD is shown in the graph as the estimated (blue circle) or measured value (red triangle). In (b) and

680 (c) the gas discharged (raw data) is shown as a function of water potential (b) and time (c). In (d) the 681 estimated water potential for every $15 \mathrm{~min}$ is shown as a function of time.
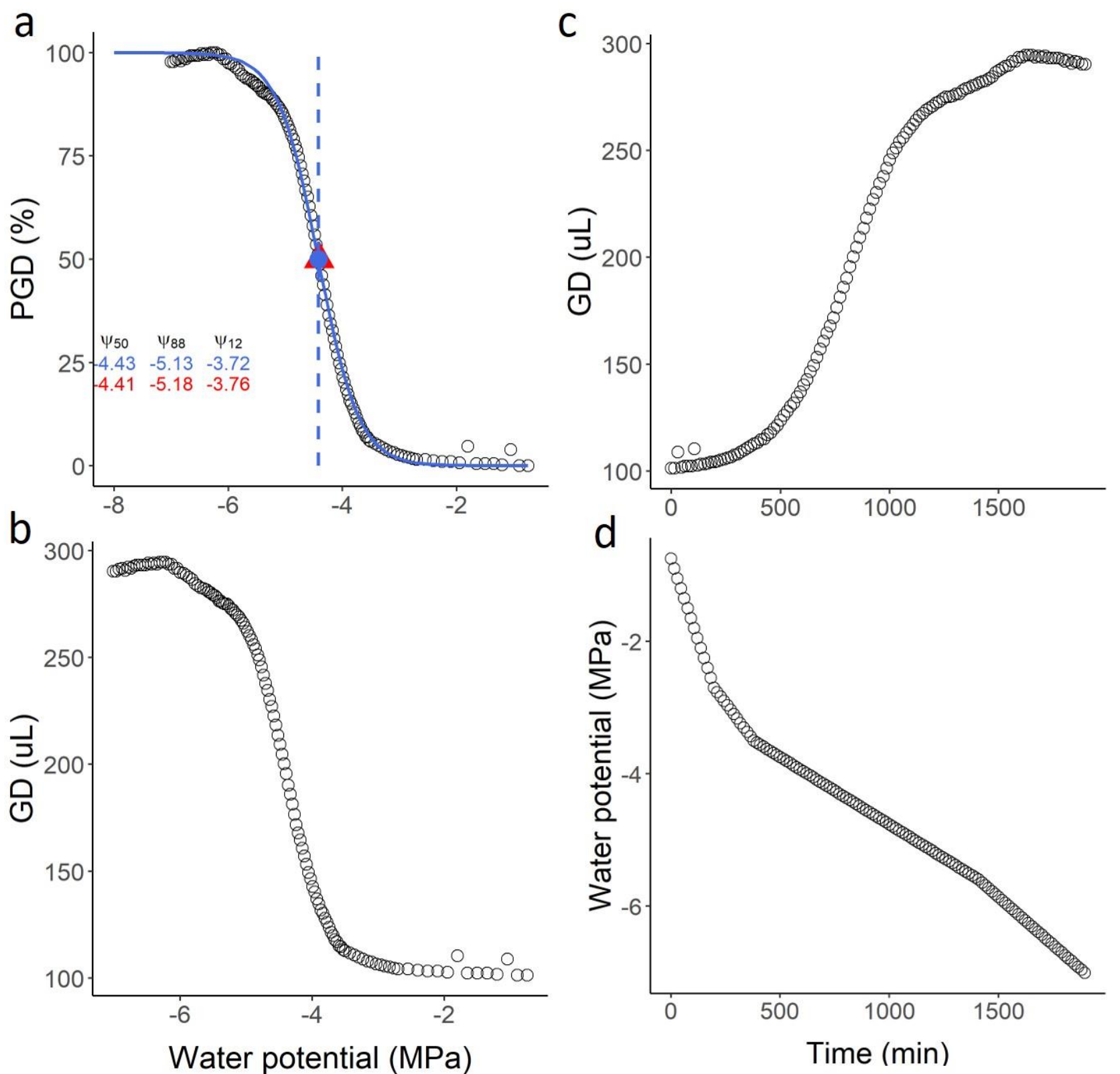